\title{
The relationship between gender and coping mechanisms with burnout events in first-year medical students
}

\author{
Rena Palupi ${ }^{1}$ and Ardi Findyartini, ${ }^{2,3}$
}

${ }^{1}$ Medicine Study Programme, ${ }^{2}$ Department of Medical Education, and ${ }^{3}$ Medical Education Center, Indonesia Medical Education and Research Institute, Faculty of Medicine, Universitas Indonesia, Central Jakarta, Indonesia

Purpose: First-year students are susceptible to experiencing burnout if the coping mechanism being used is inadequate; therefore, employing effective coping mechanisms could help students to minimize burnout. Coping mechanisms are divided into five groups: problem-focused, emotion-focused, dysfunctional coping, adaptive, and maladaptive coping. The burnout dimension includes emotional exhaustion, cynicism, and decreased academic performance that may be influenced by gender. This study aims to elaborate on the relationship between gender and coping mechanisms with burnout events in first-year medical students.

Methods: This is a cross-sectional study using a total sample of first-year students from a medical school.

Results: A total of 167 respondents (response rate 98.9\%) completed a Brief Coping Orientation to Problems Experienced Questionnaire and Maslach Burnout Inventory-Student Survey. The results showed that there was no significant relationship between gender and burnout ( $p>0.05$ ). On the contrary, maladaptive/dysfunctional coping had a significant positive correlation with emotional exhaustion $(r=0.403, p<0.001)$ and cynicism $(r=0.372, p<0.001)$. Adaptive coping had a significant negative correlation with cynicism $(r=-0.165$, $p=0.033)$ and a significant positive correlation with perception of personal accomplishment $(r=0.417, p<0.001)$.

Conclusion: In conclusion, there was no significant relationship between gender and burnout. However, maladaptive/dysfunctional coping had a positive correlation with emotional exhaustion and cynicism. On the other hand, adaptive coping had a negative correlation with cynicism and a positive correlation with perception of personal accomplishment.

Key Words: Burnout, First-year students, Sex, Coping mechanism

\section{Introduction}

Burnout is a psychological syndrome resulting from emotional exhaustion, depersonalization, and decreased performance [1]. More recent studies suggest the use of emotional exhaustion and depersonalization as two main components of burnout [2,3]. Stress could potentially cause burnout, and generally half of medical students experience stress in some forms. Medical students who experience stress tend to have lower academic grades since stress may have an impact on decision-making and working memory [4]. Research in Lebanon and Spain showed that $75 \%$ and $14.8 \%$ of medical students experienced burnout respectively [5]. This is supported by research conducted by Chang et al. [6], which stated that $55 \%$ of medical students in the first 3 years of education had high burnout levels. Major multi-
Received: April 25, 2019 • Revised: May 9, 2019 • Accepted: August 8, 2019 Corresponding Author: Ardi Findyartini (https://orcid.org/0000-0002-9601-3994) Department of Medical Education, Faculty of Medicine, Universitas Indonesia, Salemba 6 Central Jakarta, Indonesia 10430

Tel: +62.213901814 Fax: +62.213901814 email: ardi.findyartini@ui.ac.id, findyartini@yahoo.com
Korean J Med Educ 2019 Dec; 31(4): 331-342.

https://doi.org/10.3946/kjme.2019.143

eISSN: 2005-7288

(C) The Korean Society of Medical Education. All rights reserved. This is an open-access article distributed under the terms of the Creative Commons Attribution Non-Commercial License (http:// creativecommons.org/licenses/by-nc/3.0/), which permits unrestricted non-commercial use, distribution, and reproduction in any medium, provided the original work is properly cited. 
institutional studies in the United States also reported that at least half of medical students experienced burnout during medical education [7].

Studies conducted in medical students in different settings showed that medical students have high tendency of burnout [8-10]. A study in a medical school in Brazil showed that $70.6 \%$ of students had high emotional exhaustion, 52.8\% had high cynicism, and $48.7 \%$ had low academic efficacy. This prevalence is related to the learning process during medical education and transition of the learning system from teachercentered to active learning [8]. These results were consistent with the study conducted on third-year medical students in the United States, which showed that $60 \%$ of students had moderate to high levels of emotional exhaustion and above $80 \%$ had depersonalization, and also almost all of students were dissatisfied with their personal achievement [9], and with systematic review on burnout among medical students by Dyrbye et al. [10] which showed that $35 \%$ of participants had high emotional exhaustion, $26 \%$ had high depersonalization, and $31 \%$ had low level of academic efficacy. In addition, studies conducted in medical students in clinical years in Iran showed that more than $50 \%$ of students experienced a low level of burnout and around $40 \%$ of students experienced moderate to high level of burnout $[11,12]$. Study in Kashan, Iran showed that there is no significant difference of burnout incidence between gender [12]. In addition, according to study by Mahdizadeh et al. [13], there was no significant differences in depersonalization and personal achievement subscales between gender; however, the prevalence of emotional exhaustion was higher in female than in male. On the contrary, study conducted by Cecil et al. [14] showed that there was no relationship between gender and emotional exhaustion, yet the ratio of depersonalization and personal incompetence were significantly higher in male than in female.
These differences might be due to cultural differences and educational environment in each country [11].

Students will experience a transition period at each stage of their development. As such, the shift from high school to college for first-year medical students could be a contributing factor that leads to some levels of stress as they experience changes between the education system in high school compared with tertiary education [15]. In addition, the shift from preclinical to clinical year which leads to more patient exposure, increased stress, and decreased confidence is also suggested to increase the risk of burnout [16].

Medical education is a lifelong learning experience that can cause students to experience burnout if the coping mechanism used is inadequate [5]. However, adopting an appropriate coping mechanism may help students to minimize burnout [17]. As burnout is considered a product of emotional exhaustion and depersonalization, a decrease in academic performance may be seen in medical students who are struggling to cope with stress [6]. This should certainly be considered in firstyear medical students specifically, since their coping processes will often determine their academic performance in the subsequent stages of education [4].

Stress is divided into two groups: distress and eustress. Distress is damaging and unpleasant, and individuals who suffer from distress find themselves feeling afraid, anxious, uneasy, or worried. Eustress, on the other hand, is a pleasant and satisfying experience, which can increase alertness, mental readiness, and performance in individuals which then increases motivation to produce something [18]. Stress in students could be caused by their academic life, both from internal and external demands. These demands require students to adapt and overcome their problems [19].

One way for individuals to overcome stress is to employ a suitable coping mechanism. Coping mech- 
anisms include all forms of cognitive and emotional efforts carried out by individuals to overcome stressors of the presenting situation [4], and these may develop unconsciously [20]. Every student uses different coping mechanisms and, according to previous studies, finding individually appropriate coping mechanisms may reduce burnout symptoms [21]. On the other hand, poor coping mechanisms over time can develop into depressive symptoms [22]. In general, coping mechanisms are divided into two categories: problem-focused and emotion-focused. Problem-focused coping involves the individual actively changing their situation and directly confronting the stressors. Emotion-focused coping is an attempt to manage emotions resulting from stressful situations without changing the situation itself. However, these categories cannot be interpreted as being purely focused on either problems or emotions because an individual could employ a combination of both [23].

Recently, the percentage of female medical students has been increasing [24]. The research conducted by Houkes et al. [25] demonstrated that there were significant differences between men and women in terms of burnout prevalence and experience. Men tend to experience depersonalization, while burnout in women is triggered by emotional exhaustion as indicated by a decrease in accomplishment [3,25]. Therefore, it is important to provide a support system that is appropriate for students of both genders.

Given the increased number of female students and required attempts of burnout prevention in medical schools considering male and female students' characteristics and their coping mechanisms, the present authors aimed to elaborate the relationship between gender and coping mechanisms with the incidence of burnout, especially in first-year students. The hypothesis proposed in this study is as follows: There is a relationship between gender with the incidence of burnout, and there is a relationship between the coping mechanism employed with the incidence of burnout in first-year students at the Faculty of Medicine, Universitas Indonesia during the academic year of 2017/2018. This research was approved by the Research Ethics Committee of Faculty of Medicine, Universitas Indonesia (approval no., 851/UN2.F1.D1/KBK/PDP.01/ 2018).

\section{Methods}

\section{Context}

The medical faculty curriculum Universitas Indonesia 2012 edition was used for reference in this study. Undergraduate medical education at Universitas Indonesia consists of an academic stage with a minimum of seven semesters and a clinical stage over a period of four semesters (long rotations, short rotations, and pre-internship modules) [26]. Some of the learning methods at the Faculty of Medicine, Universitas Indonesia include collaborative learning, problem-based learning, interactive lectures, tutorials and practical laboratory sessions, basic clinical skills, team-based learning, specific topic discussion with question-based learning methods, e-learning, video conferences, case presentations, clinical learning experience, and field learning experience [27].

In the first year of medical education at Universitas Indonesia, namely the first and second semesters, students are required to attend obligatory courses from both university and the Health Sciences Cluster, while in the second semester students are introduced to medicine modules. As such, the first-year medical students at Universitas Indonesia have not yet been exposed to special modules in general medicine and clinical 
medicine [26], and thus are quite new to the information given, as well as the course structure and workload. The change of learning environment in medical school and more student-centered active learning approaches might become stressors for first-year medical students in the current setting.

\section{Design}

This cross-sectional study was conducted at Faculty of Medicine, Universitas Indonesia from December 2017 to October 2018 using total sampling from the inclusion criteria of active first-year medical students enrolled in the academic year of 2017/2018. The independent variables measured in this study were gender and coping mechanisms, while the dependent variable measured was burnout.

\section{Instrument}

Respondents were given two types of questionnaires, the Brief Coping Orientation to Problems Experienced (COPE) and Maslach Burnout Inventory (MBI)- Student Survey [28,29]. The Brief COPE Questionnaire was used to measure coping mechanisms. This questionnaire consists of 28 questions divided into 14 coping mechanism subscales, where each subscale consists of two questions. The Brief COPE Questionnaire is the short form of the COPE Inventory Questionnaire with some adjustments. The Brief COPE Questionnaire was calculated using the total score to see the tendency of individuals in using coping mechanisms; therefore, individuals could employ more than one type of coping mechanism [28]. The MBI-Student Survey was used to measure burnout. The questionnaire consists of 15 questions, divided into three dimensions of burnout, in which each dimension consists of five questions. As well as coping mechanisms, measurement of burnout used the total score of each dimension. The measurement of each burnout dimension using the MBI-Student Survey must be calculated separately and could not be combined into one burnout score [29].

\section{Data analysis}

The data was processed and analyzed using the IBM SPSS ver. 20.0 software (IBM Corp., Armonk, USA). Data was processed firstly using descriptive statistical tests to explore the demographic data in terms of total number and gender percentage. A bivariate analysis was then conducted to test the two proposed hypotheses. To answer the first hypothesis, the unpaired t-test with an alternative Mann-Whitney test was carried out whereas for the second hypothesis, the Pearson correlation test with an alternative Spearman correlation test was completed.

Data on all 14 coping mechanism subscales obtained from the Brief COPE Questionnaire were grouped according to Cooper et al. [30] and Meyer [31]. The classification is problem-focused (active coping, planning, use of instrumental support), emotion-focused (use of emotional support, positive reframing, acceptance, religion, humor), dysfunctional coping (venting, denial, substance use, behavioral disengagement, self-distraction, self-blame), adaptive coping (including all subscales: problem-focused and emotion-focused coping), and maladaptive coping (including all the dysfunctional coping subscales) [32].

\section{Results}

A total of 167 respondents (response rate 98.9\%) completed the questionnaires: 76 were male (45.5\%) and 91 were female (54.5\%). The Brief COPE Questionnaire and MBI-Student Survey used in this study showed good internal consistency (Cronbach's $\alpha$ of 0.722 and 0.721 ). 
Based on the Kolmogorov-Smirnov normality test conducted for all 14 coping mechanism subscales and five classifications, according to Cooper et al. [30] and Meyer [31] and in accordance with the Brief COPE Questionnaire, $\mathrm{p}<0.05$ was obtained; hence, the data from the results were described as median with minimum and maximum values. Tables 1 and 2 show the characteristics of the coping mechanisms and their classifications according to the Brief COPE Questionnaire. According to Table 1, the highest median value was related to the self-distraction and positive reframing subscale, while the lowest median value was related to

Table 1. Respondent Characteristics Based on Coping Mechanism According to Brief COPE Questionnaire $(n=167)$

\begin{tabular}{lc}
\hline \multicolumn{1}{c}{ Brief COPE Questionnaire subscales } & Median (min-max) \\
\hline Self-distraction & $7(2-8)$ \\
Active coping & $6(2-8)$ \\
Denial & $3(2-6)$ \\
Substance use & $2(2-6)$ \\
Use of emotional support & $6(2-8)$ \\
Use of instrumental support & $6(2-8)$ \\
Behavioral disengagement & $3(2-8)$ \\
Venting & $5(2-8)$ \\
Positive reframing & $7(2-8)$ \\
Planning & $6(3-8)$ \\
Humor & $5(2-8)$ \\
Acceptance & $6(4-8)$ \\
Religion & $6(2-8)$ \\
Self-blame & $5(2-8)$ \\
\hline
\end{tabular}

COPE: Coping Orientation to Problems Experienced.

Table 2. Respondent Characteristics Based on Coping Mechanism in Accordance with Brief COPE Questionnaire According to Cooper et al. [30] and Meyer [31] $(n=167)$

\begin{tabular}{lc}
\hline Brief COPE Questionnaire subscales classification & $\begin{array}{c}\text { Median } \\
\text { (min-max) }\end{array}$ \\
\hline According to Cooper et al. [30] & $19(11-24)$ \\
Problem-focused & $30(19-39)$ \\
Emotion-focused & $25(16-38)$ \\
Dysfunctional coping & \\
According to Meyer [31] & $49(33-62)$ \\
Adaptive coping & $25(16-38)$ \\
Maladaptive coping &
\end{tabular}

substance use. Table 2 shows that the highest median value was emotion-focused coping and adaptive coping. Kolmogorov-Smirnov normality test was performed on each of the burnout dimensions in accordance with the MBI-Student Survey and obtained a value of $\mathrm{p}<0.05$; therefore, the data was presented in median with minimum and maximum values. Table 3 shows that the characteristics of all three burnout dimensions according to the MBI-Student Survey and the burnout dimension with the highest median value was the perception of personal accomplishment, while the burnout dimension with the lowest median value was cynicism.

The unpaired t-test was used to evaluate the relationship between gender and emotional exhaustion because of the normal data distribution. The MannWhitney test was used to assess the relationship between gender with cynicism and perception of personal accomplishment because the data distribution was not normal. Results of the two tests showed no significant difference between gender and the three burnout dimensions (Table 4).

The second hypothesis was assessed using the Spearman correlation test to explore the relationship of coping mechanisms according to the classification of Cooper et al. [30] and Meyer [31] with all three burnout dimensions. The Spearman correlation test was used because the distribution of data was not normal. The results of several coping mechanism groups had a significant correlation with one or more dimensions of burnout, namely dysfunctional coping with emotional exhaustion,

Table 3. Respondent Characteristics Based on the Three Burnout Dimensions According to MBI-Student Survey ( $n=167)$

\begin{tabular}{lc}
\hline Burnout dimensions on MBI-Student Survey & $\begin{array}{c}\text { Median } \\
(\min -\mathrm{max})\end{array}$ \\
\hline Emotional exhaustion & $17(0-30)$ \\
Cynicism & $11(0-30)$ \\
Perception of personal accomplishment & $21(3-30)$ \\
\hline MBI: Maslach Burnout Inventory.
\end{tabular}




\begin{tabular}{lcc}
\hline Table 4. Gender and Burnout Dimensions & & \\
\cline { 2 - 3 } \multicolumn{1}{c}{ Variable } & Value & p-value \\
\hline Emotional exhaustion & $15.68 \pm 5.82$ & \\
Male $(n=76)$ & $17.14 \pm 5.86$ & 0.337 \\
Female $(n=91)$ & $11(0-28)$ & \\
Cynicism & $10(0-30)$ & 0.371 \\
Male $(n=76)$ & & \\
Female $(n=91)$ & $20.50(3-30)$ & \\
Perception of personal accomplishment & $21(7-30)$ & \\
Male $(n=76)$ & & \\
Female $(n=91)$ &
\end{tabular}

Data are presented as mean \pm standard deviation or median (min-max).

\begin{tabular}{|c|c|c|c|c|c|c|}
\hline \multirow[t]{2}{*}{ Variable } & \multicolumn{2}{|c|}{ Emotional exhaustion } & \multicolumn{2}{|c|}{ Cynicism } & \multicolumn{2}{|c|}{$\begin{array}{c}\text { Perception of personal } \\
\text { accomplishment }\end{array}$} \\
\hline & $r$ & $p$-value & $r$ & $p$-value & r & $\mathrm{p}$-value \\
\hline Problem-focused & -0.035 & 0.650 & -0.137 & 0.078 & 0.431 & $<0.001^{*}$ \\
\hline Emotion-focused & -0.112 & 0.151 & -0.162 & $0.036^{*}$ & 0.368 & $<0.001 *$ \\
\hline Dysfunctional coping & 0.403 & $<0.001^{*}$ & 0.372 & $<0.001^{*}$ & -0.080 & 0.303 \\
\hline Adaptive coping & -0.090 & 0.247 & -0.165 & $0.033^{*}$ & 0.417 & $<0.001^{*}$ \\
\hline Maladaptive coping & 0.403 & $<0.001^{*}$ & 0.372 & $<0.001^{*}$ & -0.080 & 0.303 \\
\hline
\end{tabular}

Bold type is considered statistically significant.

${ }^{*} p<0.05$.

emotion-focused and dysfunctional coping with cynicism, problem-focused and emotion-focused with the perception of personal accomplishment, adaptive coping with cynicism and perception of personal accomplishment, and maladaptive coping with emotional exhaustion and cynicism (Table 5).

\section{Discussion}

Respondents who completed the questionnaire in this study comprised 167 respondents (response rate 98.9\%) with more female (52\%) than male (48\%). This is in accordance with the population of the first-year students at Faculty of Medicine, Universitas Indonesia in the academic year of 2017/2018. These figures also align with claims that numbers of female medical students have increased proportionate to males in several countries over the past 30 years [17].

Coping mechanisms with the highest median value in this study were self-distraction and positive reframing. Self-distraction is an avoidance strategy, whereas positive reframing is based on emotion-focused coping [32,33]. Avoidance and emotional strategies both impact stress, but avoidance strategy is considered one of the risk factors that creates stress and, as previously mentioned, stress in first-year students is usually due to the transition to a tertiary institution [33]. High stress levels in first-year medical students have a positive correlation with avoidance strategy because students will either let go of the stress or stay away from stressful situations [34].

On the other hand, the coping mechanism with the lowest median value was substance use. This is in accordance with the research undertaken by Pinasthika [35], in which the coping mechanism with the lowest 
median value in the first-year student at Faculty of Medicine, Universitas Indonesia was substance use. In addition, research by Al-Dubai et al. [36] on medical students at University of Malaysia showed the same results; substance use was a coping mechanism with the lowest average value at 2.7. This result could be explained by the culture of the community influencing the coping mechanisms employed by individuals [37], and the fact that both Indonesia and Malaysia are societies with diverse cultural and spiritual values.

The burnout dimension with the highest median value was accompanied by the perception of personal accomplishment. The high result for perception of personal accomplishment showed a low level of burnout as it correlates with perception of academic performance [1]. Low perception of academic performance refers to an individual's feeling of decreased competence, productivity, and low level of success. Academic achievement, therefore, affects student behavior. Several factors influence student academic achievement, including selfconfidence and trustworthiness, which could motivate individuals to do their jobs correctly and obtain satisfactory results [38].

The burnout dimension with the lowest median value was cynicism. Cynicism is one of the negative attitudes/ emotions that is more often expressed by medical students and residents [39]. According to Testerman et al. [40], cynicism is the most prevalent factor found in medical students experiencing burnout, which decreases over time in conjunction with a doctor's career advancements that result from having developed better coping mechanisms, self-confidence, capability, and status in the health workforce team. This occurs because medical students will try to survive the demands in a medical education environment and therefore persevere with their situation. In addition, lack of experience in health services can result in medical students using cynicism as a way of controlling their environment [41].

The statistical analysis for the first hypothesis showed no significant relationship between gender with each of the burnout dimensions. Research conducted by Boni et al. [8] in first-year medical students of a medical school in Brazil also showed no significant relationship between gender and burnout $(\mathrm{p}=0.509)$. Another study among third-year medical students in obstetric and gynecology setting also showed no difference of burnout prevalence between male and female [42]. Despite this result, the average value of the female's emotional exhaustion in this present study was higher than the male's value. This might happen because females tend to suppress their emotions, while males will release negative emotions, especially when they are under pressure [3,42]. Female doctors also express more concern on the academic environment change, job dissatisfaction and decreased of professionalism in practice, compared to their male counterparts [43]. Research conducted by Houkes et al. [25] stated that emotional exhaustion could lead to burnout in female doctors. Other studies show higher burnout prevalence of female medical students than male counterparts which are due to higher impact of perceived stressor, and higher emotional responses [42].

The indifference of burnout among male and female first-year medical students in this present study might be explored further from cultural perspective. In Indonesia where collectivism is prominent [44], the display of emotional exhaustion or depersonalization tend to be discouraged. A meta-analysis conducted by Purvanova and Muros [3], suggested that cultural background, such as whether the dominant cultural value is collectivism or individualism, does not moderate the burnout levels between male and female. However, based on the Job Demand and Resources model [45], social support from family in females, and social support from colleagues in males seemed to be protective against burnout [46], and 
such support is quite common in collectivist culture. In addition, in developing country context in which individualism and masculinity are high, burnout among females can be due to high effort-low reward works whereas those among males can be caused by high effort-high reward works. In other words, while efforts for equity and recognition in workplace may lead to burnout among females, males can experience burnout due to high competition [47]. In the current era of Indonesia where roles of gender become increasingly balanced, both female and male medical students might experience similar competitive environment and the level of burnout becomes indifferent, despite tendency of more emotional exhaustion among female first-year medical students.

The Spearman correlation test used to prove the second hypothesis in this study showed a significant positive correlation between dysfunctional/maladaptive coping with emotional exhaustion and cynicism, adaptive coping (problem-focused, emotion-focused) with perception of personal accomplishment, and a significant negative correlation between adaptive coping (emotionfocused) with cynicism.

Dysfunctional coping and maladaptive coping had a significant positive correlation with emotional exhaustion in which the two groups include the same coping mechanism subscales, namely venting, denial, substance use, behavioral disengagement, self-distraction, and self-blame. Emotional exhaustion in the sufferer is reflected by excessive emotions and work-related exhaustion, which presents as physical fatigue and a feeling of being "drained", both psychologically and emotionally. Passive responses, such as avoidance, equivocation, and self-withdrawal, could affect emotional control because the underlying situation may remain unresolved which then causes stress. Avoidance and self-withdrawal do not resolve the situation, and both responses are positively related with emotional exhaustion [48].

Similar with emotional exhaustion, dysfunctional coping and maladaptive coping had a significant positive correlation with cynicism. Cynicism is a negative attitude involving cognitive, affective, and behavioral aspects, which progressively produce negative impacts on an individual's wellbeing. Cynicism is positively related with an ineffective coping mechanism, meaning that when facing a difficult challenge, a very cynical person will stop trying to achieve their initial goals [49].

On the contrary, emotion-focused and adaptive coping had a significant negative correlation with cynicism. All emotion-focused subscales are part of adaptive coping, thereby the significant part of the coping mechanism related to cynicism is emotion-focused, including the use of emotional support, positive reframing, acceptance, religion, and humor. Cynicism is negatively correlated with effective coping mechanisms, such as seeking instrumental and emotional support, planning, reframing, and turning to religion [49]. Successful emotional regulation contributes to improved performance and wellbeing and can reduce burnout and increase pleasant emotions. Emotional dissociation could help medical students to overcome their emotions in the short-term, but in the long-term it could cause a decrease in empathy and an increase in cynicism [50].

Adaptive coping, which includes problem-focused (active coping, planning, and use of instrumental support) and emotion-focused techniques, had a significant positive correlation with the perception of personal achievement. Adaptive coping can prevent burnout symptoms in which individuals will face problems by being proactive and address their behavior. A low perception of personal achievement reflects feelings of dissatisfaction and loss of responsibility in performing tasks. According to Cumbe et al. [48], 
individuals with high perceptions of personal achievement are characterized by high values of adaptive coping, which is in accordance with this study.

This study is limited as it was conducted only in one medical school, therefore the results obtained may not be generalized to other institutions. However, considering the large sample used in this study and similar circumstances presenting for first-year medical students needing to cope in the transition period in all medical schools, the results of this study may remain relevant for other institutions.

In conclusion, overall, the coping mechanism found to have the highest median value in this study was selfdistraction and positive reframing, while the lowest median value was substance use. In addition, based on the classification of Cooper et al. [30] and Meyer [31], the coping mechanisms with the highest median value were emotion-focused coping and adaptive coping. On the other hand, the burnout dimension with the highest median value was the perception of personal accomplishment, while the lowest median value was cynicism. In addition, this study showed no significant relationship between gender and the incidence of burnout in first-year students at Faculty of Medicine, Universitas Indonesia in the academic year of 2017/2018. Yet it was found that dysfunctional/maladaptive coping had a significant, intermediate positive correlation with emotional exhaustion and cynicism. Also, adaptive coping (emotion-focused) had a significantly weak negative correlation with cynicism and a significant, intermediate positive correlation with perception of personal accomplishment.

The results of this study can be used by students to understand the relationship between coping mechanisms and burnout during their first year of medical school. These results demonstrate the existing burnout dimensions and can provide students with ways to adopt appropriate coping mechanisms to avoid burnout during learning activities. For medical schools, the results of this study could be used as a basis to facilitate student support programs on burnout prevention and demonstrate how to harness appropriate coping mechanisms, especially for first-year students, in addition to raising students' awareness of this matter. Finally, the results of this study could be used as the basis for future research on a broader scale regarding coping mechanisms and burnout at all stages of education.

\section{ORCID:}

Rena Palupi: https://orcid.org/0000-0002-2535-6543; Ardi Findyartini: https://orcid.org/0000-0002-9601-3994 Acknowledgements: We would like to thank all firstyear medical students of academic year 2017/2018 who participated voluntarily in this study. We would like to acknowledge the support of Faculty of Medicine Universitas Indonesia for providing the publication grant for the present study. We would also like to extend our gratitude to Dr Retno Asti Werdhani who provided feedback on the statistical analysis and Ms Daniar Setyorini who facilitated the data collection.

Funding: None.

Conflicts of interest: No potential conflict of interest relevant to this article was reported.

Author contributions: RP conducted the study, completed data collection and analysis, and contributed to manuscript development. AF designed the study, completed data analysis, and led the manuscript development.

\section{References}

1. Maslach C, Jackson SE, Leiter MP. Maslach Burnout 
Inventory. Palo Alto, USA: Consulting Psychologists Press; 1981.

2. Maslach C, Leiter MP. Early predictors of job burnout and engagement. J Appl Psychol. 2008;93(3):498-512.

3. Purvanova RK, Muros JP. Gender differences in burnout: a meta-analysis. J Vocat Behav. 2010;77(2):168-185.

4. Schiller JH, Stansfield RB, Belmonte DC, et al. Medical students' use of different coping strategies and relationship with academic performance in preclinical and clinical years. Teach Learn Med. 2018;30(1):15-21.

5. Fares J, Al Tabosh H, Saadeddin Z, El Mouhayyar C, Aridi H. Stress, burnout and coping strategies in preclinical medical students. N Am J Med Sci. 2016; 8(2):75-81.

6. Chang E, Eddins-Folensbee F, Coverdale J. Survey of the prevalence of burnout, stress, depression, and the use of supports by medical students at one school. Acad Psychiatry. 2012;36(3):177-182.

7. Ishak W, Nikravesh R, Lederer S, Perry R, Ogunyemi D, Bernstein C. Burnout in medical students: a systematic review. Clin Teach. 2013;10(4):242-245.

8. Boni RA, Paiva CE, de Oliveira MA, Lucchetti G, Fregnani JH, Paiva BS. Burnout among medical students during the first years of undergraduate school: prevalence and associated factors. PLoS One. 2018;13(3):e0191746.

9. Amir E, Kumari S, Olivetta U, Mansoor M. Burnout and depression among medical students at Historically Black Colleges and Universities (HBCU) hospital system. Int J Psychol Behav Anal. 2018;4:151.

10. Dyrbye LN, West CP, Satele D, et al. Burnout among U.S. medical students, residents, and early career physicians relative to the general U.S. population. Acad Med. 2014;89(3):443-451.

11. Ebrahimi S, Atazadeh F. Medical students' occupational burnout and its relationship with professionalism. J Adv Med Educ Prof. 2018;6(4):162-167.

12. Sepehrmanesh Z, Ahmadvand A, Akasheh G, Saei, R.
P02-119: prevalence of burnout in senior medical students. Eur Psychiatry. 2010;25(Suppl 1):723.

13. Mahdizadeh M, Vafaei A, Taghipour A, Esmaeily H, Mahdizadeh SM, Ebrahimipour H. Occupational burnout and factors affecting the work environment among family physicians of Khorasan Razavi. Q J Occup Med. 2014;5(4):42-51.

14. Cecil J, McHale C, Hart J, Laidlaw A. Behaviour and burnout in medical students. Med Educ Online. 2014; $19: 25209$

15. Santrock JW. Perkembangan anak. Ilth ed. Jakarta, Indonesia: Erlangga; 2008.

16. Dyrbye LN, Thomas MR, Harper W, et al. The learning environment and medical student burnout: a multicentre study. Med Educ. 2009;43(3):274-282.

17. Dunn LB, Iglewicz A, Moutier C. A conceptual model of medical student well-being: promoting resilience and preventing burnout. Acad Psychiatry. 2008;32(1):44-53.

18. Kariv D, Heiman T. Task-oriented versus emotionoriented coping strategies: the case of college students. Coll Stud J. 2005;39(1):72-89.

19. Selye H. The stress of my life: a scientist's memoirs. 2nd ed. New York, USA: Van Nostrand Reinhold; 1979.

20. Somaiya M, Kolpakwar S, Faye A, Kamath R. Study of mechanisms of coping, resilience and quality of life in medical undergraduates. Indian J Soc Psychiatry. 2015; 31(1):19-28.

21. Gan Y, Shang J, Zhang Y. Coping flexibility and locus of control as predictors of burnout among Chinese college students. Soc Behav Pers. 2007;35(8):1087-1098.

22. Fresco DM, Williams NL, Nugent NR. Flexibility and negative affect: examining the associations of explanatory flexibility and coping flexibility to each other and to depression and anxiety. Cogn Ther Res. 2006;30(2): 201-210.

23. Litman JA. The COPE inventory: dimensionality and relationships with approach-and avoidance-motives and 
positive and negative traits. Pers Individ Differ. 2006; 4l(2):273-284.

24. Burton KR, Wong IK. A force to contend with: the gender gap closes in Canadian medical schools. CMAJ. 2004; 170(9):1385-1386.

25. Houkes I, Winants Y, Twellaar M, Verdonk P. Development of burnout over time and the causal order of the three dimensions of burnout among male and female GPs: a three-wave panel study. BMC Public Health. $2011 ; 11: 240$.

26. Tridjaja B, Findyartini A, Wahid MH, et al. Curriculum of Undergraduate Medical Program Faculty of Medicine Universitas Indonesia 2012. Jakarta, Indonesia: Fakultas Kedokteran Universitas Indonesia; 2012.

27. Harden RM, Sowden S, Dunn WR. Educational strategies in curriculum development: the SPICES model. Med Educ. 1984;18(4):284-297.

28. Carver CS. You want to measure coping but your protocol's too long: consider the brief COPE. Int J Behav Med. 1997;4(1):92-100.

29. Maslach C, Jackson SE, Leiter MP, Schaufeli WB, Scwab RL. Maslach Burnout Inventory manual. 4th ed. Menlo Park, USA: Mind Garden Inc.; 2016.

30. Cooper C, Katona C, Orrell M, Livingston G. Coping strategies and anxiety in caregivers of people with Alzheimer's disease: the LASER-AD study. J Affect Disord. 2006;90(1):15-20.

31. Meyer B. Coping with severe mental illness: relations of the Brief COPE with symptoms, functioning, and wellbeing. J Psychopathol Behav Assess. 2001;23(4):265277.

32. Su XY, Lau JT, Mak WW, et al. A preliminary validation of the Brief COPE instrument for assessing coping strategies among people living with HIV in China. Infect Dis Poverty. 2015;4:41.

33. Mohammed MM. Stress and coping among international medical students at Lithuanian University of Health
Sciences (LUHS) [dissertation]. Kaunas, Lithuania: Lithuanian University of Health Sciences; 2016.

34. Margareth A, Bassols AM, Carneiro BB, et al. Stress and coping in a sample of medical students in Brazil. Arch Clin Psychiatry. 2015;42(1):1-5.

35. Pinasthika A. Relationships between gender and place of origin with coping mechanism of first-year medical students in undergraduate medical program Faculty of Medicine Universitas Indonesia academic year 2015/2016 [undergraduate thesis]. Jakarta, Indonesia: Universitas Indonesia; 2016.

36. Al-Dubai SA, Al-Naggar RA, Alshagga MA, Rampal KG. Stress and coping strategies of students in a medical faculty in Malaysia. Malays J Med Sci. 2011;18(3): 57-64.

37. Tweed RG, White K, Lehman DR. Culture, stress, and coping: internally-and externally-targeted control strategies of European Canadians, East Asian Canadians, and Japanese. J Cross Cult Psychol. 2004;35(6):652-668.

38. Hu Q, Schaufeli WB. The factorial validity of the Maslach Burnout Inventory-Student Survey in China. Psychol Rep. 2009;105(2):394-408.

39. Karimi A, Saadatmand Z. The relationship between selfconfidence with achievement based on academic motivation. Kuwait Chapter Arab J Bus Manag Rev. 2014; $4(1): 210-215$.

40. Testerman JK, Morton KR, Loo LK, Worthley JS, Lamberton $\mathrm{HH}$. The natural history of cynicism in physicians. Acad Med. 1996;71(10):43-45.

41. Batley NJ, Nasreddine Z, Chami A, Zebian D, Bachir R, Abbas HA. Cynicism and other attitudes towards patients in an emergency department in a middle eastern tertiary care center. BMC Med Educ. 2016;16:36.

42. Worly B, Verbeck N, Walker C, Clinchot D, Keder L, Steinauer J. Gender differences in medical students' experience of burnout, perceived stress, and empathic concern. Obstet Gynecol. 2017;130:61S 
43. LaFaver K, Miyasaki JM, Keran CM, et al. Age and sex differences in burnout, career satisfaction, and well-being in US neurologists. Neurology. 2018;91(20):e1928el941.

44. Hofstede G. Culture's consequences: comparing values, behaviors, institutions, and organizations across nations. 2nd ed. Thousand Oaks, USA: Sage Publications; 2001.

45. Bakker AB, Demerouti E. The job demands-resources model: state of the art. J Manag Psychol. 2007;22(3): 309-328.

46. Verweij H, van der Heijden FM, van Hooff ML, et al. The contribution of work characteristics, home characteristics and gender to burnout in medical residents. Adv Health Sci Educ Theory Pract. 2017;22(4):803-818.
47. Devonish D. Gender, effort-reward imbalance at work, and burnout: findings from a developing Caribbean country. Gend Manag. 2017;32(6):441-452.

48. Cumbe VF, Pala AN, Palha AJ, et al. Burnout syndrome and coping strategies in Portuguese oncology health care providers. Rev Psiquiatr Clin. 2017;44(5):122-126.

49. Sendal L, Krzyzaniak P, Raczynska A, Atroszko PA. The mediating effect of stress coping between cynical hostility and perceived stress: preliminary findings. Poznan, Poland: Młodzi Naukowcy; 2016.

50. Doulougeri K, Panagopoulou E, Montgomery A. (How) do medical students regulate their emotions? BMC Med Educ. 2016;16(1):312. 